\title{
RESEARCH
}

Open Access

\section{Ulcer metastasis? Anatomical locations of recurrence for patients in diabetic foot remission}

Brian J. Petersen ${ }^{1 *} \mathbb{D}$, Gary M. Rothenberg ${ }^{2}$, Priti J. Lakhani ${ }^{3}$, Min Zhou', David R. Linders ${ }^{1}$, Jonathan D. Bloom', Katherine A. Wood ${ }^{1}$ and David G. Armstrong ${ }^{4}$

\begin{abstract}
Background: The "cancer analogy" is powerful for communicating risk to and organizing care for patients with diabetic foot syndrome. One potentially underappreciated similarity between cancer and foot ulcers is that both can recur at anatomical locations distinct from the primary occurrence, albeit with different physiological mechanisms. Few studies have characterized the location of diabetic foot ulcer recurrence, and these have been limited by considering only the first recurrent wound following a recent-healed wound. We therefore characterized the anatomical locations at which diabetic foot ulcers are likely to recur considering multiple wounds during follow-up and the locations of all prior wounds documented in the participant's history.

Methods: We completed a secondary analysis of existing data from a 129 participant multi-center study of participants in diabetic foot remission. The primary outcome was plantar foot ulceration, and each participant was followed for 34 weeks or until withdrawing consent, allowing characterization of all wounds occurring. We stratified the anatomical locations of wounds prior to the trial by the following outcome categories during the trial: no recurrence, recurrence to the same anatomical location, recurrence to a different anatomical location on the same foot, and recurrence to the contralateral foot.
\end{abstract}

Results: A large percentage (48\%) of wounds recurred to the contralateral foot, and the proportion of subsequent foot ulcer to the contralateral limb was largely unaffected by the anatomical location of foot ulcer prior to the study. Only $17 \%$ of prior diabetic foot ulcers were followed by recurrence to the same anatomical location. Rates of recurrence remained high during treatment of a wound ( 0.41 foot ulcer/ulcer-year). Participants had documented wounds to 2.2 distinct anatomical locations on average, and more than $60 \%$ of participants had wounds to more than one plantar location by the end of the study.

Conclusions: Given the significant morbidity, mortality, and resource utilization associated with foot ulcer recidivism, quality and evidenced-based preventive care is essential. Our results better characterize the burden of recurrence and to what anatomy recurrence is most likely. These insights may benefit providers and patients alike for the provision of high-quality preventive care thereby resulting in reduced morbidity, mortality, and cost.

Trial registration: The study providing the data for this secondary analysis was registered on ClinicalTrials.gov (NCT02647346) on January 6, 2016. The study was retrospectively registered.

Keywords: Diabetic foot, Diabetic foot ulcer, Recurrence, Prevention, Epidemiology

\footnotetext{
* Correspondence: authors@podiography.org

${ }^{1}$ Podimetrics Inc, Somerville, MA, USA

Full list of author information is available at the end of the article
}

(c) The Author(s). 2020 Open Access This article is distributed under the terms of the Creative Commons Attribution 4.0 International License (http://creativecommons.org/licenses/by/4.0/), which permits unrestricted use, distribution, and reproduction in any medium, provided you give appropriate credit to the original author(s) and the source, provide a link to the Creative Commons license, and indicate if changes were made. The Creative Commons Public Domain Dedication waiver (http://creativecommons.org/publicdomain/zero/1.0/) applies to the data made available in this article, unless otherwise stated. 


\section{Background}

Since Armstrong and colleagues highlighted that the five year mortality rates associated with foot ulceration and lower extremity amputation exceed several common cancers [1], including those of the breast and prostate, the "cancer analogy" has become an effective education and communication tool for practitioners and patients alike. The analogy has helped convey the seriousness of diabetic foot syndrome and has helped guide conversations regarding expectations and outcomes. Consider a diabetic foot ulcer (DFU) as a "malignancy" of the foot, and "wound hospice" as a viable alternative to conventional treatment. Diabetic foot "remission" is a key concept for helping patients and caregivers appreciate the ongoing risk of recurrence after a wound has healed.

The cancer analogy is appropriate: both cancer and DFU are associated with significant mortality [2] and reduced quality of life [3]; both have substantial burden of illness, confronting patients and caregivers alike with time-consuming and challenging treatment regimens [4]; both are commonly refractory and chronic [5]; both are associated with recidivism [6]; and both result in considerable resource utilization and cost [7].

One potentially underappreciated similarity between DFU and cancer is that both can recur at anatomical locations distinct from the primary occurrence, albeit with different underlying physiological mechanisms. Few studies have characterized the location of DFU recidivism relative to the location of previous wounds [8-11]. These studies found that only a minority of wounds (between 19 and 37\%) recur at the same anatomical location as a patient's most recently-healed DFU. In fact, a 2017 paper by Ornheholm and colleagues [8] reported that $38 \%$ of recurrent plantar forefoot DFU (excluding minor digits) are to the contralateral limb.

The risk of recurrence to the contralateral limb is additionally reflected in research reporting rates of lower extremity amputations (LEA). In the year following a LEA, approximately $10 \%$ of patients have a second amputation of the contralateral limb [12-16], and as many as $50 \%$ have amputation of the contralateral limb within five years following a primary amputation [13, 17-19]. While LEA rates overall have improved over recent decades [20], this survey of the literature suggests that secondary LEA rates to the contralateral limb have not meaningfully improved over several generations.

Unfortunately, the existing literature on the location of DFU recurrence is limited in two important ways. First, these studies considered only the first recurrence and did not report on all locations of previous DFU. Second, existing data were not stratified granularly by anatomical location. We therefore report on a secondary analysis of existing data in order to better characterize the anatomical locations at which DFU have recurred in study participants. These data may inform improved preventive practice, leading to better patient outcomes.

\section{Methods}

We abstracted data from a multicenter trial in 129 participants (NCT02647346), which assessed the accuracy of a remote temperature monitoring floor mat (Podimetrics RTM System; Somerville, MA) [21]. This trial was conducted across seven outpatient sites in the United States. Participants were required to have a history of DFU, be ambulatory, and have documented ankle-brachial index exceeding 0.5 in the absence of palpable pedal pulses. Patients with unhealed DFU, active Charcot foot disease, end-stage renal disease, or immunosuppressive disease were not eligible for participation. The study was conducted in accordance with the Helsinki Declaration and was approved by multiple institutional review boards as required by the individual enrollment centers.

Each participant was followed under standard diabetic foot care for 34 weeks or until withdrawing consent. Upon enrollment, investigators conducted a chart review to characterize the anatomical locations of previous DFU for each participant. During the study, participants were instructed to contact the investigators if a lesion (defined as an anatomical area which has suffered injury or damage) or DFU was noted during daily self-exam. All lesions and wounds were treated consistent with standard practice. Additionally, each participant was contacted by phone during months 2 and 4 of the trial and asked if he or she noticed any change to either foot. At the conclusion of the trial, each participant was evaluated in clinic for any lesions and wounds, and study staff completed a final chart review to confirm that all relevant DFU were captured as study outcomes.

The primary outcome during the trial was recurrence of plantar DFU with non-acute etiology. We adopt the definitions of the International Working Group on the Diabetic Foot [22] and define a "foot ulcer" as a break of the skin of the foot penetrating through the epidermis and at a minimum through part of the dermis. "Healed" was defined as macroscopic epithelialization. We define a "recurrent foot ulcer" as a foot ulcer developing after healing of a previous foot ulcer, located at any site on either foot. Participants were followed through the end of the trial or until withdrawing consent, thus allowing investigators to characterize incidence of all plantar DFU occurring during participation. During the trial, 37 participants had a total of 53 recurrent plantar DFU (0.63 DFU/participant-year) to all limbs.

We stratified DFU outcomes during the trial into the following categories: 
1. the participant remained in diabetic foot remission, i.e. had no recurrent foot ulcers during the trial

2. the participant had a recurrent foot ulcer to the same anatomical location as a prior wound

3. the participant had a recurrent foot ulcer to a different anatomical location on the same foot as a prior wound

4. the participant had recurrent foot ulcer to the contralateral foot

Additionally, we calculated the number of distinct locations of DFU (considering each digit, metatarsal head, arch, and heel as a distinct location) occurring to each participant through the end of the trial, including those documented in the patient's medical records prior to study enrollment.

\section{Results}

In this secondary analysis of existing data, we reported on the breakdown of the anatomical locations of DFU both prior to and during the trial. During the 34 week trial, 92 participants $(71.3 \%)$ remained in diabetic foot remission. In those who did have a recurrent foot ulcer during the trial (28.7\%), $48 \%$ of DFU recurred to the contralateral foot, $35 \%$ of DFU recurred to a different anatomical location on the same foot, and $17 \%$ recurred at the same location as a previous DFU.

Table 1 shows the distribution of DFU locations on a per-participant basis. Prior to the trial, participants were most likely to have DFU history at the hallux (57\%), lesser digits (34\% combined to the third, fourth, and fifth toes), second digit (28\%), 1st metatarsal head (26\%), and heel (7\%). During participation, the most common locations for recurrence were 1st metatarsal head (41\%), hallux (32\%), and 5th metatarsal head (16\%).

Table 1 Participants with Foot Ulcers Stratified by Anatomical Location Prior to and during the Study

\begin{tabular}{lll}
\hline Anatomical Location & \multicolumn{2}{c}{ Percentage of Participants with DFU at Location } \\
\cline { 2 - 3 } & Prior to Study & During Study \\
\hline Hallux & $56.6 \%$ & $32.4 \%$ \\
2nd Digit & $27.9 \%$ & $8.1 \%$ \\
1st Metatarsal Head & $25.6 \%$ & $40.5 \%$ \\
3rd Digit & $13.2 \%$ & $10.8 \%$ \\
5th Metatarsal Head & $12.4 \%$ & $16.2 \%$ \\
3rd Metatarsal Head & $12.4 \%$ & $8.1 \%$ \\
5th Digit & $11.6 \%$ & $5.4 \%$ \\
2nd Metatarsal Head & $11.6 \%$ & $8.1 \%$ \\
4th Digit & $8.5 \%$ & $0.0 \%$ \\
4th Metatarsal Head & $7.0 \%$ & $5.4 \%$ \\
Heel & $7.0 \%$ & $8.1 \%$ \\
\hline
\end{tabular}

Figure 1 shows the distribution of anatomicallydistinct DFU locations for participants through the end of the study. Upon study completion, participants had a history of DFU to $2.2+/-2.0$ distinct anatomical locations on average. More than $60 \%$ of participants had DFU to more than one plantar location, and one participant had history of DFU to nine distinct anatomical locations.

Whereas Table 1 shows the percentage of participants with wounds to different locations, Figs. 2 and 3 show the anatomical locations of wounds prior to the trial and subsequent outcomes during the trial as a percentage of total number of wounds documented. Medial forefoot DFU were disproportionately more likely to recur at the same location (17.7\%) than other anatomical locations, while lateral forefoot DFU were most likely to be followed by a DFU at another location on the same foot (25.9\%). Approximately half of recurrent DFU in cases where the previous DFU was to the first metatarsal head were followed by contralateral recurrence $(52 \%, 17 / 33)$. Similarly, nearly half $(49 \%, 17 / 35)$ of previous hallux DFU were followed by contralateral recurrence during the trial. The proportion of subsequent DFU to the contralateral limb was largely unaffected by the anatomical location of DFU prior to the study.

A total of four foot ulcer recurrences occurred to two participants being treated for an unhealed DFU that occurred during the trial (0.41 DFU/ulcer-year). Two of these recurred to the contralateral limb during treatment (0.20 DFU/ulcer-year).

\section{Discussion}

Our results suggest that DFU to patients in diabetic foot remission frequently occur in anatomically-distinct locations, including a large percentage to the contralateral foot. We reported a larger proportion of recurrence to the contralateral foot than Orneholm and colleagues [8], with $48 \%$ of the DFU incident during the 34 week trial recurring to the limb opposite a prior DFU. Only $17 \%$ of prior DFU were followed by recurrence to the same anatomical location during follow up. Additionally, patients undergoing treatment for unhealed wounds maintain high recurrence rates despite increased clinical attention during treatment: we report a high rate of incidence to participants being treated for an unhealed DFU that occurred during the trial (0.41 DFU/ulcer-year).

These data have important implications for preventive care. Major risk factors for DFU, which include peripheral neuropathy and peripheral arterial disease, commonly affect the entirety of both limbs. Given this fact and our results, preventive care should be provisioned for both feet and not only on areas of previous ulceration or concern. Accordingly, patients and caregivers should be educated on the elevated risk to both feet and 


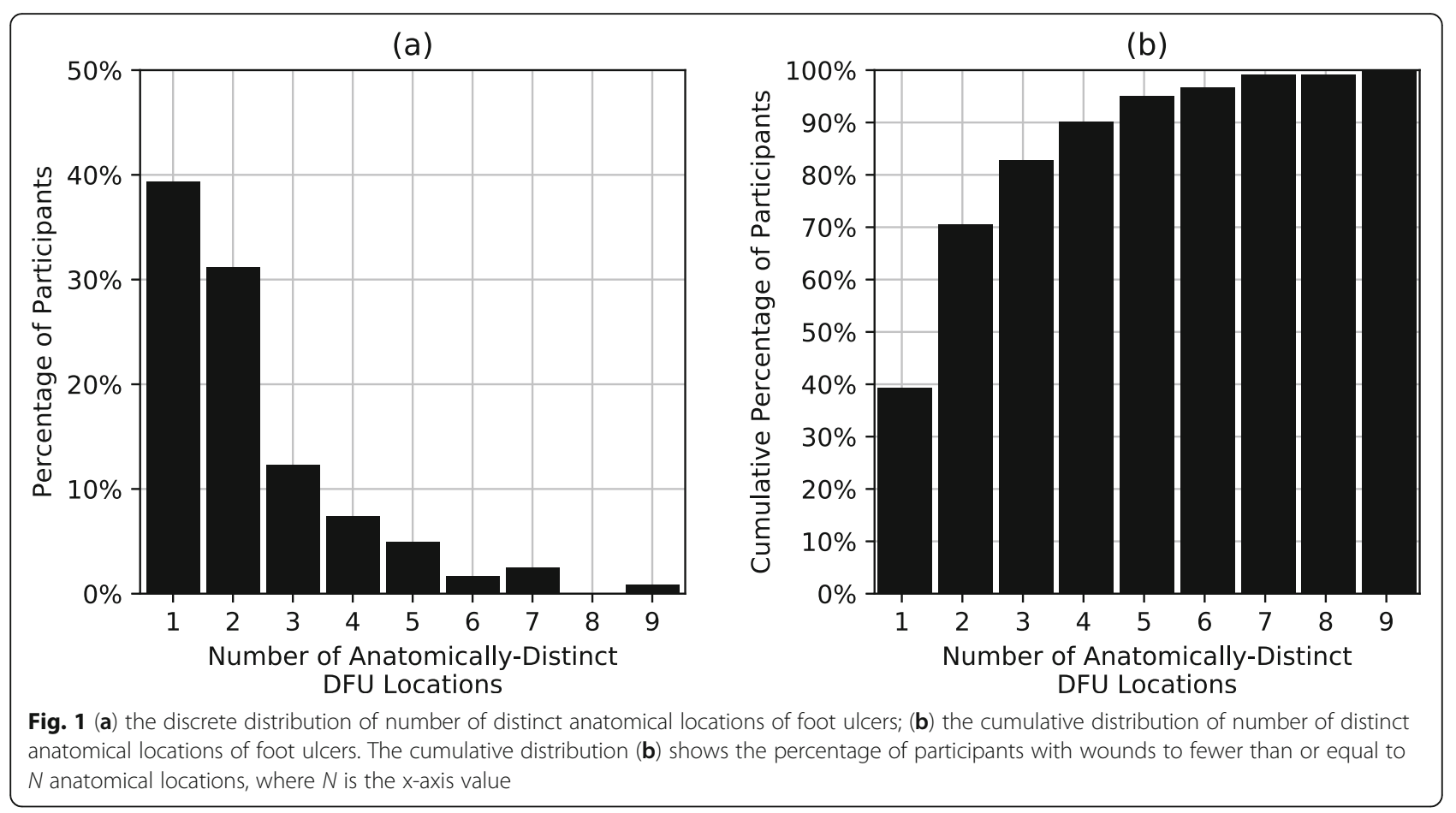

instructed to thoroughly examine the entirety of both feet daily for discoloration, callus, blisters, fissures, and other pre-ulcerative findings.

Given our findings of high incidence to the foot without a wound during treatment for a DFU, providers should also be cognizant of the risk to both limbs and let this insight guide practice. For example, while treating a DFU, providers should remain attentive to foot without a wound, which may be predisposed to elevated risk not only chronically due to neuropathy and arterial disease but also acutely due to gait deviation and pressure redistribution secondary to treatment of the wounded foot.

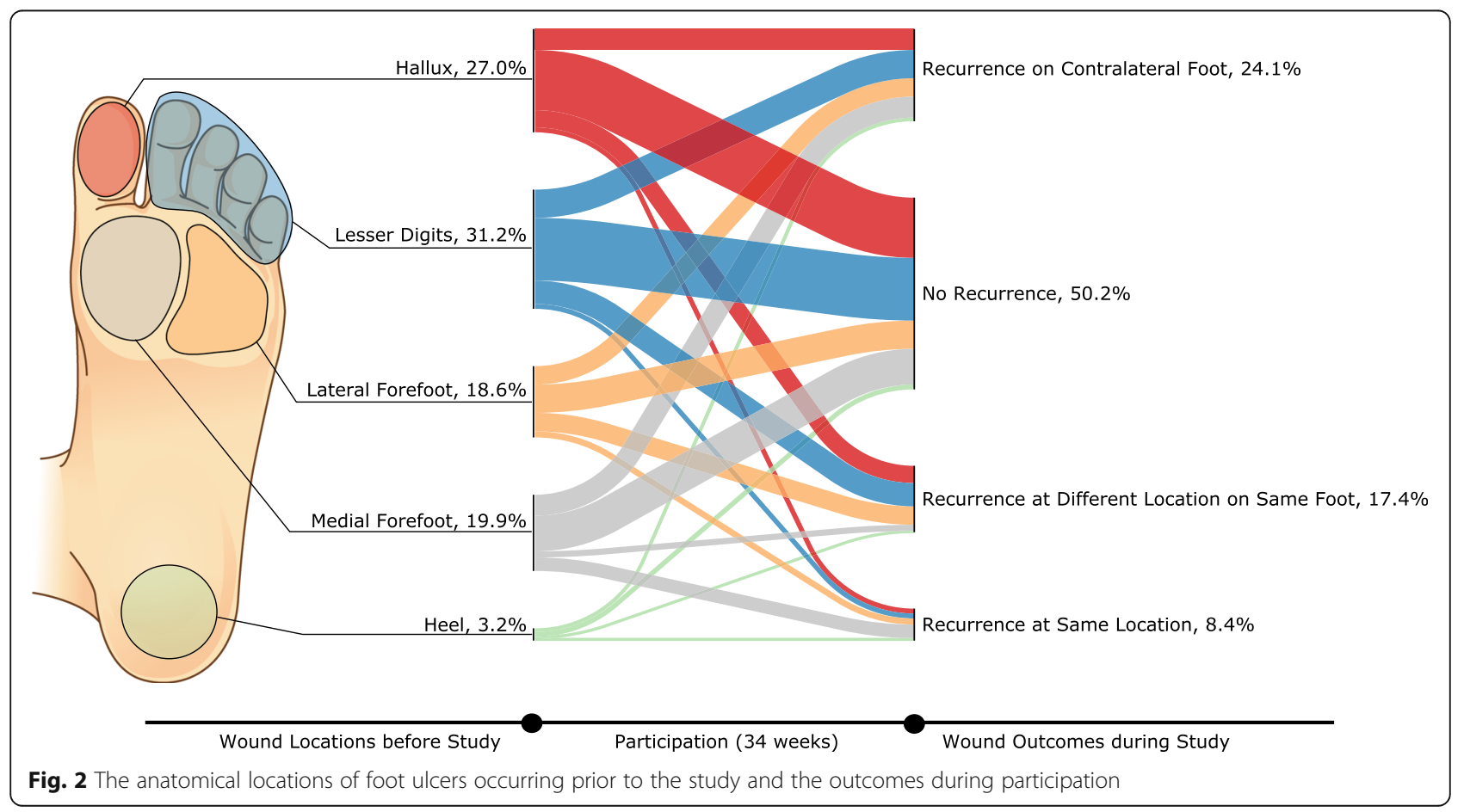




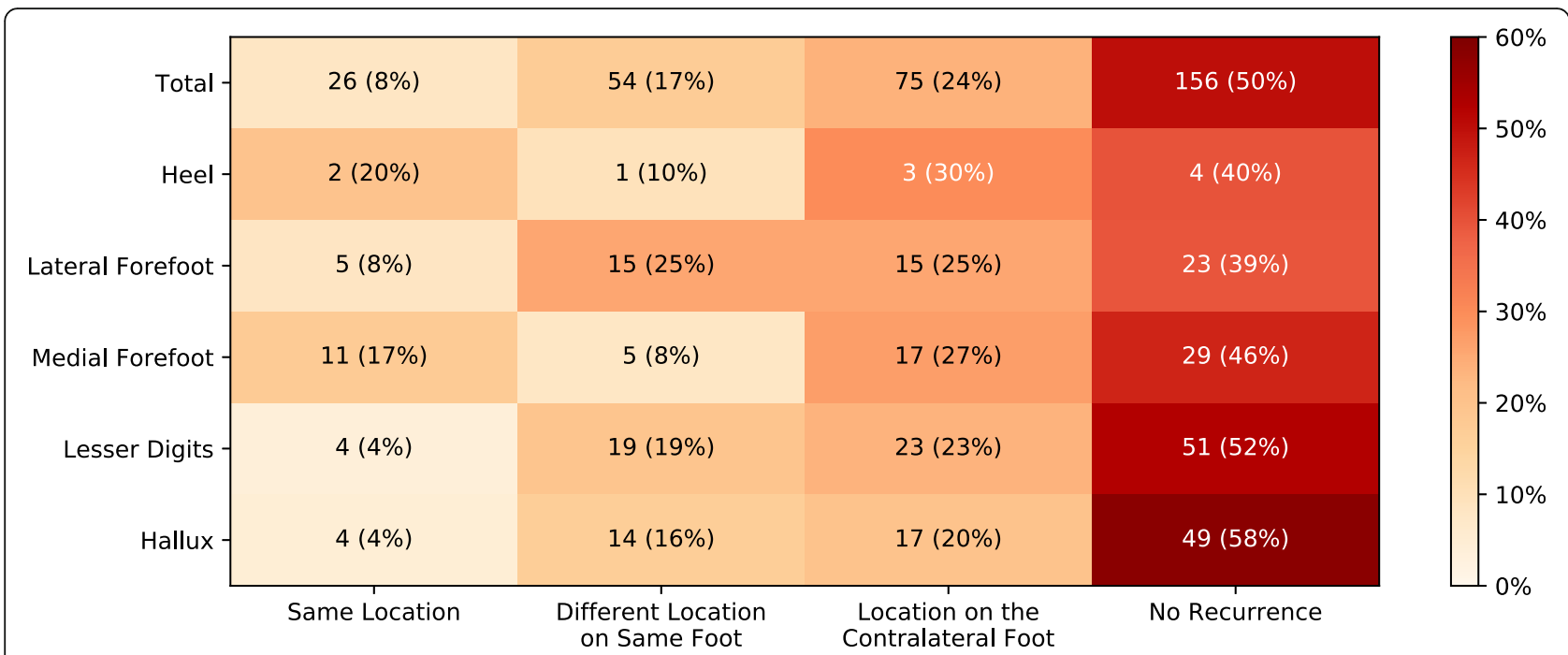

Fig. 3 The anatomical locations of foot ulcers occurring prior to the study and the outcomes during participation

Additionally, our results may be helpful for interpreting the data from emerging telemedicine technologies for the diabetic foot, such as once-daily foot temperature monitoring [23]. These new monitoring modalities have been shown to improve outcomes for those in diabetic foot remission [24-26], allowing for early and targeted intervention much like mammography and colonoscopy for breast and colon cancer, respectively. A better understanding of patient risk, including the anatomical locations of recurrence, can provide additional context for those utilizing such emerging modalities for the diabetic foot in remission.

Unlike previous investigations, one benefit of our current effort is that we considered the anatomical locations of all DFU documented in each participant's history, not just the most recent. This design has allowed us to fully characterize the burden of recurrence for those patients with diabetic foot syndrome, which is sobering. A majority of participants $(60 \%)$ had a history of DFU at two distinct anatomical locations prior to the trial, and nearly one in five participants had history of DFU to four or more plantar locations on the feet. Prior to the trial, more than half of participants $(57 \%)$ had a history of DFU to the hallux, and more than a quarter had a previous DFU to the 1st metatarsal head. During the trial, recurrence was less likely to the digits, possibly as a result of prior amputation of distal anatomy, which was highly prevalent among the participants [21].

\section{Conclusion}

In our data, DFU to participants in diabetic foot remission frequently occurred to anatomy different from the locations of previously-healed wounds. A large percentage (48\%) of wounds recurred to the contralateral foot, and only $17 \%$ of prior DFU were followed by recurrence to the same anatomical location. Rates of recurrence remained high during treatment (0.41 DFU/ulcer-year). Overall participants had documented wounds to 2.2 distinct anatomical locations on average, and more than $60 \%$ of participants had DFU to more than one plantar location.

Given the significant morbidity, mortality, and resource utilization associated with DFU recidivism, quality and evidenced-based preventive care is essential. Our results better characterize the burden of recurrence and also may inform improved understanding of the anatomical locations of recurrence. These insights may improve practice of preventive care for those in diabetic foot remission, thereby reducing morbidity, mortality, and cost.

\section{Abbreviations}

DFU: Diabetic foot ulcer; LEA: Lower extremity amputation

\section{Acknowledgements}

Nicole J Neff contributed to the copyediting and assembly of this manuscript.

\section{Authors' contributions}

BJP. concept and design, analysis of data, interpretation of results, preparation and review of manuscript. GMR. interpretation of results, preparation and review of manuscript. PJL. interpretation of results, preparation and review of manuscript. MZ. analysis of data; interpretation of results, preparation and review of manuscript. DRL. interpretation of results, preparation and review of manuscript. JDB. preparation and review of manuscript. KAW. preparation and review of manuscript. DGA. interpretation of results, preparation and review of manuscript. All authors contributed to critically revise the manuscript and have given final approval of the version to be published.

\section{Authors' information}

Not applicable.

\section{Funding}

Podimetrics, Inc. funded the study on which this secondary analysis was based as its sole sponsor, including providing the study devices and financial support to the clinical sites for study operations. 
The secondary analysis constituting the current study did not receive any specific grant from any funding agency in the public, commercial or not-forprofit sectors.

\section{Availability of data and materials}

All data generated or analyzed during this study are included in this published article.

\section{Ethics approval and consent to participate}

The study on which this secondary analysis of existing data was based was approved by the New England Institutional Review Board and local review boards for VA Phoenix (Phoenix, AZ), VA Long Beach (Long Beach, CA), VA Miami (Miami, FL), and Greenville Health System (Greenville, SC). Each participant freely gave informed consent prior to participation in the study.

\section{Consent for publication}

Not applicable.

\section{Competing interests}

BJP, DRL, MZ, KAW, and JDB are employees of Podimetrics Inc, a private company which designed and manufactured the study device and sponsored the study on which this research is based.

GMR and PJL are consultant Medical Directors at Podimetrics, Inc

DGA is a member of the Scientific Advisory Board of Podimetrics, Inc.

\section{Author details}

${ }^{1}$ Podimetrics Inc, Somerville, MA, USA. ${ }^{2}$ University of Michigan Medical School, Ann Arbor, MI, USA. ${ }^{3}$ Einstein Healthcare Network, Philadelphia, PA, USA. ${ }^{4}$ Keck School of Medicine, University of Southern California, California, Los Angeles, USA.

Received: 25 October 2019 Accepted: 6 January 2020

Published online: 13 January 2020

\section{References}

1. Armstrong DG, Wrobel J, Robbins JM. Guest editorial: are diabetes-related wounds and amputations worse than cancer? Int Wound J. 2007:4(4):286-7.

2. Brennan MB, Hess TM, Bartle B, Cooper JM, Kang J, Huang ES, et al. Diabetic foot ulcer severity predicts mortality among veterans with type 2 diabetes. J Diabetes Complicat. 2017:31(3):556-61.

3. Beattie AM, Campbell R, Vedhara K. What ever I do its a lost cause. The emotional and behavioural experiences of individuals who are ulcer free living with the threat of developing further diabetic foot ulcers: a qualitative interview study. Health Expect. 2014:17(3):429-39.

4. Nabuurs-Franssen MH, Huijberts MSP, Nieuwenhuijzen Kruseman AC, Willems J, Schaper NC. Health-related quality of life of diabetic foot ulcer patients and their caregivers. Diabetologia. 2005;48(9):1906-10.

5. Searle A, Gale L, Campbell R, Wetherell M, Dawe K, Drake N, et al. Reducing the burden of chronic wounds: prevention and management of the diabetic foot in the context of clinical guidelines. J Health Serv Res Policy. 2008;13(Suppl 3):82-91.

6. Armstrong DG, Boulton AJM, Bus SA. Diabetic foot ulcers and their recurrence. N Engl J Med. 2017;376(24):2367-75.

7. Chan B, Cadarette S, Wodchis W, Wong J, Mittmann N, Krahn M. Cost-ofillness studies in chronic ulcers: a systematic review. J Wound Care. 2017; 26(Sup4):S4-14.

8. Orneholm H, Apelqvist J, Larsson J, Eneroth $\mathrm{M}$. Recurrent and other new foot ulcers after healed plantar forefoot diabetic ulcer. Wound Repair Regen. 2017;25(2):309-15.

9. Galea AM, Springett K, Bungay H, Clift S, Fava S, Cachia M. Incidence and location of diabetic foot ulcer recurrence. Diabetic Foot Journal. 2009:12(4):181-6.

10. Peters EJG, Armstrong DG, Lavery LA. Risk factors for recurrent diabetic foot ulcers. Diabetes Care. 2007;30(8):2077-9.

11. Khalifa WA. Risk factors for diabetic foot ulcer recurrence: a prospective 2year follow-up study in Egypt. Foot. 2018:35:11-5.

12. Ebskov B, Josephsen P. Incidence of reamputation and death after gangrene of the lower extremity. Prosthetics Orthot Int. 1980;4(2):77-80.

13. Baddeley RM, Fulford JC. A trial of conservative amputations for lesions of the feet in diabetes mellitus. Br J Surg. 1965;52:38-43.

14. Font-Jiménez I, Llaurado-Serra M, Roig-Garcia M. De los Mozos-Perez B, Acebedo-Urdiales S. retrospective study of the evolution of the incidence of non-traumatic lower-extremity amputations (2007-2013) and risk factors of reamputation. Prim Care Diabetes. 2016:10(6):434-41.

15. Shah SK, Bena JF, Allemang MT, Kelso R, Clair DG, Vargas L, et al. Lower Extremity Amputations [Internet]. Vol. 47, Vascular and Endovascular Surgery. 2013. p. 608-13. Available from: https://doi.org/10.1177/ 1538574413503715

16. Glaser JD, Bensley RP, Hurks R, Dahlberg S, Hamdan AD, Wyers MC, et al. Fate of the contralateral limb after lower extremity amputation. J Vasc Surg. 2013;58(6):1571-7.e1.

17. Silbert S. Amputation of the lower extremity in diabetes mellitus; a followup study of 294 cases. Diabetes. 1952;1(4):297-9.

18. Izumi Y, Satterfield K, Lee S, Harkless LB. Risk of reamputation in diabetic patients stratified by limb and level of amputation: a 10-year observation. Diabetes Care. 2006;29(3):566-70.

19. Mazet $R$ Jr. The geriatric amputee. Artif Limbs. 1967:11(2):33-41.

20. Li Y, Burrows NR, Gregg EW, Albright A, Geiss LS. Declining rates of hospitalization for nontraumatic lower-extremity amputation in the diabetic population aged 40 years or older: U.S., 1988-2008. Diabetes Care. 2012; 35(2):273-7.

21. Frykberg RG, Gordon IL, Reyzelman AM, Cazzell SM, Fitzgerald RH, Rothenberg GM, et al. Feasibility and efficacy of a smart mat Technology to predict development of diabetic plantar ulcers. Diabetes Care. 2017:40(7):973-80.

22. Bus SA, van Netten JJ, Lavery LA, Monteiro-Soares M, Rasmussen A, Jubiz Y, et al. IWGDF guidance on the prevention of foot ulcers in at-risk patients with diabetes. Diabetes Metab Res Rev. 2016;32(Suppl 1):16-24.

23. Basatneh R, Najafi B, Armstrong DG. Health sensors, smart home devices, and the internet of medical things: an opportunity for dramatic improvement in Care for the Lower Extremity Complications of diabetes. J Diabetes Sci Technol. 2018;12(3):577-86.

24. Lavery LA, Higgins KR, Lanctot DR, Constantinides GP, Zamorano RG, Armstrong DG, et al. Home monitoring of foot skin temperatures to prevent ulceration. Diabetes Care. 2004;27(11):2642-7.

25. Lavery LA, Higgins KR, Lanctot DR, Constantinides GP, Zamorano RG, Athanasiou KA, et al. Preventing diabetic foot ulcer recurrence in high-risk patients: use of temperature monitoring as a self-assessment tool. Diabetes Care. 2007;30(1):14-20.

26. Armstrong DG, Holtz-Neiderer K, Wendel C, Mohler MJ, Kimbriel HR, Lavery $\mathrm{LA}$. Skin temperature monitoring reduces the risk for diabetic foot ulceration in high-risk patients. Am J Med. 2007:120(12):1042-6.

\section{Publisher's Note}

Springer Nature remains neutral with regard to jurisdictional claims in published maps and institutional affiliations.

Ready to submit your research? Choose BMC and benefit from:

- fast, convenient online submission

- thorough peer review by experienced researchers in your field

- rapid publication on acceptance

- support for research data, including large and complex data types

- gold Open Access which fosters wider collaboration and increased citations

- maximum visibility for your research: over $100 \mathrm{M}$ website views per year

At BMC, research is always in progress.

Learn more biomedcentral.com/submission 